\title{
Micro-bioreactor array for controlling cellular microenvironments $\dagger$
}

\author{
Elisa Figallo, $\ddagger^{a}$ Christopher Cannizzaro, $\ddagger^{b}$ Sharon Gerecht, $\dagger^{b}$ Jason A. Burdick, ${ }^{c}$ Robert Langer, ${ }^{b}$ \\ Nicola Elvassore ${ }^{* a}$ and Gordana Vunjak-Novakovic* ${ }^{* d}$
}

\author{
Received 3rd January 2007, Accepted 24th April 2007 \\ First published as an Advance Article on the web 15th May 2007 \\ DOI: $10.1039 / b 700063 d$
}

High throughput experiments can be used to spatially and temporally investigate the many factors that regulate cell differentiation. We have developed a micro-bioreactor array (MBA) that is fabricated using soft lithography and contains twelve independent micro-bioreactors perfused with culture medium. The MBA enables cultivation of cells that are either attached to substrates or encapsulated in hydrogels, at variable levels of hydrodynamic shear, and with automated image analysis of the expression of cell differentiation markers. The flow and mass transport in the MBA were characterized by computational fluid dynamic (CFD) modeling. The representative MBA configurations were validated using the $\mathrm{C} 2 \mathrm{C} 12$ cell line, primary rat cardiac myocytes and human embryonic stem cells (hESCs) (lines H09 and H13). To illustrate the utility of the MBA for controlled studies of hESCs, we established correlations between the expression of smooth muscle actin and cell density for three different flow configurations.

\section{Introduction}

The cellular microenvironment, also termed a "cell niche", controls and regulates stem cell fate. ${ }^{1,2}$ Historically, the term "niche" was used to describe the stem cell location, ${ }^{3}$ the components of the microenvironment surrounding the cells, and the biochemical or electrical signals produced by the support cells. ${ }^{4,5}$ The niche functions as a physical anchor and generates a number of extrinsic factors that control cell fate. From an engineering perspective, we may define the stem cell niche as a microenvironment with multiple regulatory factors, molecular and physical, that change in space and time and govern stem cell behavior. Replicating in vitro the "cell niche" normally found in vivo would likely help understand and ultimately control the signaling pathways that regulate cell fate, and help utilize the potential of stem cells in regenerative medicine. ${ }^{6-8}$ Each of the standard cell culture methods have advantages but also limitations. Well plates are easy to use, but they are suitable only for studies of molecular factors and cannot provide physical regulatory factors. Also, well plates operate essentially batch-wise, with the composition of medium constantly changing between the two medium replacements. In contrast, cell culture bioreactors can provide perfusion of medium, ${ }^{9-13}$ control medium composition and thereby support the cultivation of high cell densities, including

\footnotetext{
${ }^{a}$ Department of Chemical Engineering, University of Padova, Italy. E-mail: nicola.elvassore@unipd.it

${ }^{b}$ Harvard-MIT for Health Sciences and Technology, Massachusetts Institute of Technology, Cambridge, MA, USA

${ }^{c}$ Department of Bioengineering, University of Pennsylvania, Philadelphia, PA 19104, USA

${ }^{d}$ Department of Biomedical Engineering, Columbia University, 1210 Amsterdam Avenue, MC 8904, New York, NY 10027, USA.

E-mail:gv2131@columbia.edu

$\dagger$ This paper is part of a special issue 'Cell and Tissue Engineering in

Microsystems' with guest editors Sangeeta Bhatia (MIT) and

Christopher Chen (University of Pennsylvania).

\# Equally contributing authors.
}

cultures of human embryonic stem cells (hESCs) ${ }^{14,15}$ and embryoid bodies. ${ }^{16}$ However, the operating volumes of bioreactors are larger than those of well plates, which is a serious limitation in studies involving the use of expensive media components. Microscale approaches can potentially overcome both of these limitations. ${ }^{13,17-24}$

Soft lithography techniques, pioneered by Whitesides and colleagues, ${ }^{25}$ are inexpensive and relatively facile, and have found widespread application in bioseparation processes, ${ }^{26,27}$ micropatterning of cells and biomaterials, ${ }^{28,29}$ cell-based assays, ${ }^{30,31}$ and cell culture devices. ${ }^{32-34}$ Early applications of cell culture devices relate to hepatocytes where microscale features provide a reasonable analog for the microstructure of the liver. ${ }^{35}$ More recently, microfluidic platforms of interconnected chambers have been proposed as "living cell arrays" for studying gene expression ${ }^{36}$ and co-culture. ${ }^{21}$

In the existing microfluidic systems for stem cell differentiation, the individual chambers are not independent, as medium is distributed across one or more columns/rows of the array, leading to the cross-talk between the cells. By enclosing each chamber, this problem may be avoided, although it now becomes more difficult to control the individual chambers without resorting to an integrated valving system. ${ }^{13}$ Additionally, direct access to the cell growth surface makes it possible to apply coatings and uniformly seed the cells.

Here we propose micro-bioreactor arrays (MBAs) designed to address some of the limitations discussed above. MBAs are a hybrid between a bioreactor (represented by each individual culture well) and a microfluidic device (represented by the system providing independent flow of medium to each individual culture well). The MBA device combines the advantages of multi-well plates (small volume, high throughput, independent culture wells) and perfusion bioreactors (steady-state conditions, enhanced mass transport, application of physical signals). To take advantage of imaging compatibility of this device, we also developed an automated image 
analysis routine that enables fast and unbiased analysis of nuclear and cytoplasmic differentiation markers.

We use the term "micro-bioreactor" because 12 culture wells, $3.5 \mathrm{~mm}$ in diameter $\times 2 \mathrm{~mm}$ deep, are located within a device that is the size of a microscope slide $(26 \times 76 \mathrm{~mm})$. Two different configurations of the MBA were developed based on computational flow modeling and analysis of mass transport: MBA-bottom inlet/outlet (BIO; medium flows directly over the substrate with attached cells) and MBA-middle inlet/outlet (MIO; medium flows above the plane with the cells in a monolayer or encapsulated in hydrogel). These MBA configurations were validated for cultivations of the $\mathrm{C} 2 \mathrm{C} 12$ cell line, primary rat cardiac myocytes and hESCs (lines H09 and H13). To illustrate the utility of the MBA for studies of hESCs, we established quantitative correlations between the density of hESCs differentiating into vascular lineages and the expression of smooth muscle actin.

\section{MBA design}

The design requirements for the MBA were: (1) high throughput experimentation with independent conditions for each bioreactor well, and low consumption of reagents and cells; (2) cultivation of cells in both the 2D setting (attached to a substrate) and three dimensional (3D) setting (encapsulated in hydrogel); (3) reproducible steady-state conditions in terms of cell density, medium composition, levels of oxygen and $\mathrm{pH}$, flow regime, hydrodynamic shear and transport rates; (4) accurate spatial-temporal control of the cell environment; (5) in situ quantitative analysis of cell proliferation and differentiation, by automated image analysis of differentiation markers.

Fig. 1 shows the design of the system. The MBA is fabricated using soft lithography techniques, has the footprint of a standard microscope slide, and contains twelve independent culture wells. The constituent elements are shown in a top view (Fig. 1A): inlet (top, shown in red) and outlet (bottom shown in yellow) ports, culture wells (orange), gas exchangers (microfluidic channels between the inlet ports and the culture wells), stream splitters (divide single inlet/outlet streams by four), and the fluidic channels (connecting the inlets, gas exchangers, culture wells and outlets).

The microfluidic channels are $100 \mu \mathrm{m}$ high by $100 \mu \mathrm{m}$ wide. Gas exchangers (20 mm total length) are designed to equilibrate the oxygen level and $\mathrm{pH}$ in each of the three inlet streams of culture medium. A splitter then divides each of these streams into four equal parts, resulting in a total of twelve inlet streams leading into the twelve culture wells. In this way, each MBA has three inlet/outlet ports and three four-way splitters directing flow to the $4 \times 3$ array of culture wells. The total length of the fluid paths leading into each well is equivalent, and the pressure drop and velocity field are thus also equivalent for each of the culture wells.

The diameter of each culture well $(3.5 \mathrm{~mm})$ was selected to provide a small volume of medium (e.g., $30 \mu \mathrm{l}$ for $\mathrm{BIO}$ configuration) and the surface area $\left(\sim 10 \mathrm{~mm}^{2}\right)$ large enough for a statistically significant number of cells to adhere $\left(\sim 10^{3}\right)$. With standard soft lithography, the height of the microbioreactor chamber would be restricted to the thickness of the
A
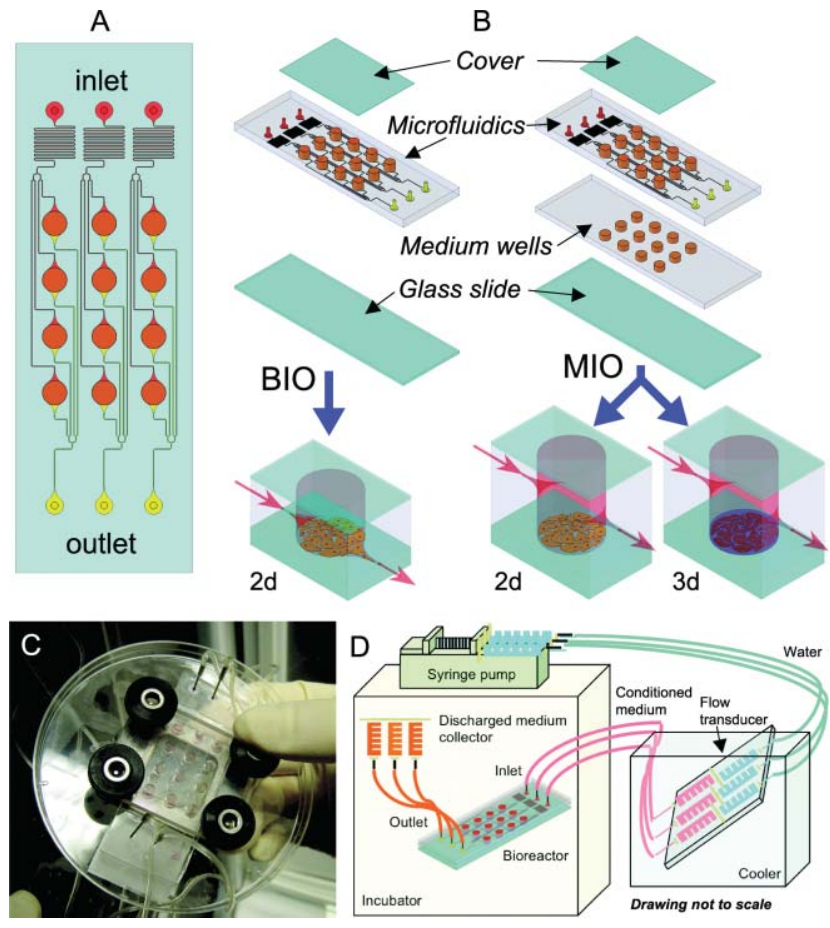

Fig. 1 MBA design. (A) The micro-bioreactor wells $(3.5 \mathrm{~mm}$ in diameter) are arranged in a $4 \times 3$ array $(8 \mathrm{~mm}$ vertical and $7 \mathrm{~mm}$ horizontal center-to-center spacing). The microfluidic channels are $100 \mu \mathrm{m}$ wide by $100 \mu \mathrm{m}$ high and deliver between $0.1-2.0 \mathrm{ml}$ of medium per day per bioreactor well. Each of three inlets delivers medium (red) through the flow transducers to four wells (orange) via microfluidic channels. Waste medium exits each bioreactor via a separate set of channels (yellow). The devices are assembled from layers of poly(dimethylsiloxane) (PDMS) and glass attached via plasma treatment of both surfaces. (B) Two configurations were used. A bottom inlet/outlet (BIO) configuration (left) consists of a glass slide, a microfluidic layer, and a gas permeable membrane cover. A middle inlet/outlet (MIO) configuration (right) has an additional layer of PDMS with an array of wells inserted between the microfluidic layer and the slide. The BIO and MIO configurations accommodate the 2D cultivation of cells attached to a substrate (glass with or without additional coating); a thin layer of a photopolymerizable hydrogel in the base of the MIO configuration wells allows 3D cultivation. (C) Image of a single MBA with compression frame and fluidic connections. (D) Experimental setup. MBAs and medium collectors are placed in an incubator for temperature $\left(37{ }^{\circ} \mathrm{C}\right)$ and gas composition $\left(5 \% \mathrm{CO}_{2}\right)$ control. Medium reservoirs are maintained external to the incubator in an ice bath. The syringes are affixed to a plate that allows a second set of syringes filled with water to actuate plungers via an external low-flow multi-channel syringe pump.

photoresist, generally between $50 \mu \mathrm{m}$ and $200 \mu \mathrm{m}$. For our applications, this would result in three constraints: (i) limited access to the cell chamber for surface coating, cell seeding, and analysis, (ii) insufficient medium hold-up within the bioreactor chamber for cell survival without perfusion, and (iii) relatively high shear stresses for medium perfusion. For these reasons, we chose to work with an "open" microfluidic device where each chamber may be individually addressed (i.e., open) and has a height equal to that of device. A removable gas permeable membrane is used to enclose each chamber during experimentation. 
Fig. 1B shows the two configurations of the MBA device that were used in the present study. Configuration BIO consists of a single layer of poly(dimethylsiloxane) (PDMS) with replica molded microfluidic channels and is perforated with an array of culture wells. The microfluidic channels are placed on the glass slide, such that the medium enters and exits the well at the bottom surface and flows directly over the attached cells (Fig. 1B, left).

Configuration MIO has an additional layer of PDMS, which (i) increases the depth of the culture well and (ii) presents medium inlets and outlets in the middle plane (Fig. 1B, right). As in the $\mathrm{BIO}$ configuration, the cells are cultured in a monolayer formed on the glass slide (with or without coating), with the main difference being a substantial reduction in exposure to hydrodynamic shear by culturing the cells outside the main plane of fluid flow. To accommodate the cultivation of hESCs in a 3D setting, a thin layer of photopolymerizing hydrogel may be added to the base of the wells in this configuration. Since cells in vivo are typically surrounded on all sides by extracellular matrix components, ${ }^{24,37,38}$ this approach more closely mimics the in vivo environment. The compressive stiffness of HA hydrogel can be varied from $0.5-50 \mathrm{kPA}$, by varying the concentration of HA and the level of crosslinking. The hydrogel layer experiences only a small level of shear stress; it is thin enough (typically $200-500 \mu \mathrm{m}$ ) to avoid major constraints in mass transfer and changes in flow patterns, yet thick enough for the cells to mimic some aspects of their native 3D environment.

The use of thin hydrogels and medium perfusion provides favorable conditions for encapsulation of hESCs in photopolymerizable hydrogels. First, the high surface to volume ratio of hydrogel in the wells $\left(20-50 \mathrm{~cm}^{2} \mathrm{~cm}^{-3}\right)$ allows for thin film (minimal light attenuation with depth) assumptions to be made. Also, medium perfusion efficiently removes any residual non-polymerized reactants.

The gas permeable membrane that seals each of the MBA chambers is held in place with a compression frame consisting of an aluminium frame, a clear polycarbonate cover, and four thumb screws (Fig. 1C). The experimental set-up is designed to assure steady state conditions during the dynamic culture. Each column of wells receives medium from one $10 \mathrm{ml}$ syringe placed on ice (to minimize protein degradation) and is controlled with a syringe pump (Fig. 1D). Each outlet tube from the MBA was connected to a discharge medium collector placed $\sim 20 \mathrm{~cm}$ above the device, all within the incubator.

\section{Operating conditions: flow and mass transport}

To characterize the operating conditions of the MBA, we evaluated the two configurations shown in Fig. 2B, in order to establish predictive flow and mass transport correlations for optimizing the conditions in the MBA. The large flow resistance within the $100 \mu \mathrm{m}$ microfluidic channels ensures equivalence of flow conditions in the twelve individual microbioreactor wells of the MBA. At the entrance and exit of each well, the channel width is gradually increased to reach the diameter of the well $(1: 35$ ratio of diameters, $1: 20$ ratio of heights), and minimize abrupt changes in fluid velocity. With the gradual change in the effective channel size and the dominance of viscous forces on the microscale, the flow field should remain laminar and uniform. Fluid flow was evaluated by solving the steady-state Navier-Stokes equations for incompressible fluid. The $3 \mathrm{D}$ solutions for the $\mathrm{BIO}$ and $\mathrm{MIO}$ configurations show that the pressure distribution within the chambers are uniform, with $>99 \%$ of the pressure drop occurring at the inlet and the outlet to the chamber (Fig. 2 A-B). The uniformity of flow is further supported by the magnitude of the fluid velocity, which is very low throughout the culture well. In the BIO configuration, the fluid velocity in a plane $50 \mu \mathrm{m}$ above the cell culture surface is less than $10 \mu \mathrm{m} \mathrm{s}^{-1}$ over the entire surface area, and less than $3 \mu \mathrm{m} \mathrm{s}{ }^{-1}$ for $75 \%$ of the area (Fig. 2C). At these low flow rates, the shear stress is $<0.01 \mathrm{dyn} \mathrm{cm}^{-2}$. In the MIO configuration, the fluid velocity and shear stress are even lower $\left(<0.01 \mu \mathrm{m} \mathrm{s}^{-1}\right.$, $<0.0001$ dyn $\mathrm{cm}^{-2}$, Fig. 2D). These simulations show that both configurations operate at very low-shear, but with clear differences (two orders of magnitude) in the values of fluid velocity and hydrodynamic shear.

A semi-quantitative analysis of mass transport for each of the two flow regimes was performed for two representative molecules: oxygen (the most critical small molecule) and albumin (a molecule representative of large growth factors) (Fig. 2E-G). As expected, the thickness of the boundary layer was lower for the BIO configuration, and the mass transport coefficient was higher for oxygen than for albumin and higher for the BIO than for the MIO configuration. The Peclet number $(P e)$ is a measure of the relative contribution of convection with respect to diffusion. At $P e>1$, mass transport is dominated by convection, a situation associated with efficient exchange of nutrients and metabolites between the cells and culture media. In contrast, the dominance of diffusion $(P e<1)$ may be associated with the accumulation of cell-secreted factors, hypoxia and changes in $\mathrm{pH}$. The $P e$ numbers for the MIO configuration strongly suggest that diffusion dominates mass transport, whereas for the BIO configuration, convection plays a much larger role with diffusion only important for small molecules (oxygen) and negligible for large molecules. This analysis demonstrates the flexibility of an MBA approach for quantitative experimentation. Through simple regulation of flow rate, chamber height, and inlet/outlet configuration, a wide range of mass transport regimes are attainable.

\section{Validation of the MBA for representative cell types: C2C12, rat cardiac myocytes and hESCs}

To validate the utility of the MBA for controlled studies of cell growth and differentiation, we selected three model systems that are representative of the envisioned applications of this device: C2C12 myoblast cell line ( $\mathrm{MIO}, \mathrm{BIO})$, primary cardiac myocytes derived from neonatal rat hearts (MIO, BIO), and hESCs (MIO). These cell types have well defined differentiation markers, and we have extensive experience in their use in various $2 \mathrm{D}$ and $3 \mathrm{D}$ configurations. ${ }^{39-42}$ Fig. 3 shows representative growth and differentiation data, at the time points characteristic for each cell type.

C2C12 cells readily adhered to the glass micro-bioreactor surface and formed spatially uniform monolayers with 
MBA BIO
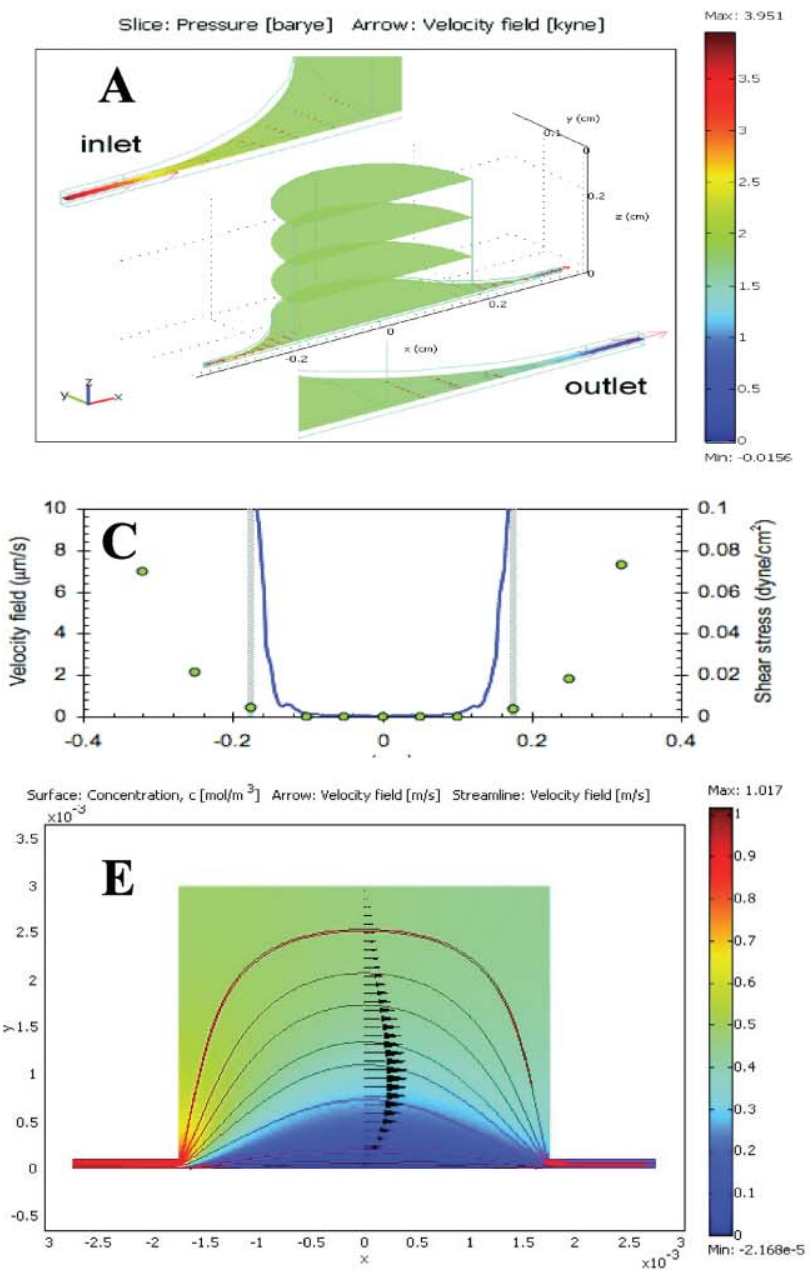

\section{MBA MIO}
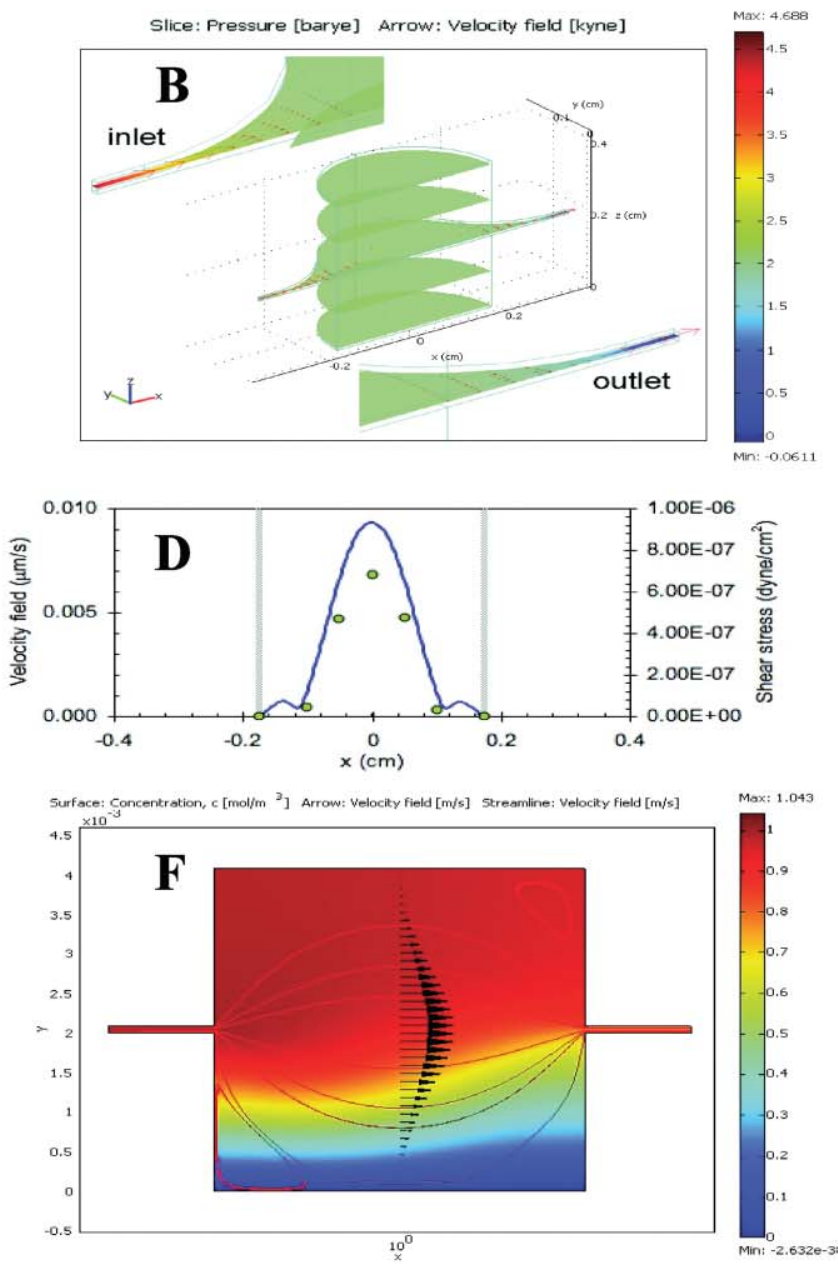

\begin{tabular}{|c|c|c|c|c|}
\hline Molecule & $\begin{array}{l}\text { Average velocity } \\
(\mathrm{m} / \mathrm{s})\end{array}$ & $\begin{array}{c}\text { Diffusion coefficient } \\
\text { D }\left(\mathrm{cm}^{2} / \mathrm{s}\right)\end{array}$ & $\begin{array}{c}\text { Re number } \\
(100 \mu \mathrm{m} \text { above the cells })\end{array}$ & $\begin{array}{c}\text { Pe number } \\
(100 \mu \mathrm{m} \text { above the cells })\end{array}$ \\
\hline \multicolumn{5}{|l|}{ MBA BIO } \\
\hline Oxygen & $1.4 \cdot 10^{-5}$ & $3.3 \cdot 10^{-5}$ & $3.7 \cdot 10^{-3}$ & $4.2 \cdot 10^{-1}$ \\
\hline Albumin & $1.4 \cdot 10^{-5}$ & $7.0 \cdot 10^{-7}$ & $3.7 \cdot 10^{-3}$ & $2.0 \cdot 10^{1}$ \\
\hline \multicolumn{5}{|l|}{ MBA MIO } \\
\hline Oxygen & $3.3 \cdot 10^{-9}$ & $3.3 \cdot 10^{-5}$ & $8.9 \cdot 10^{-7}$ & $1.0 \cdot 10^{-4}$ \\
\hline Albumin & $3.3 \cdot 10^{-9}$ & $7.0 \cdot 10^{-7}$ & $8.9 \cdot 10^{-7}$ & $4.7 \cdot 10^{-3}$ \\
\hline
\end{tabular}

Fig. 2 Flow and mass transport conditions in the MBA. (A, B) Pressure distribution (color map) and velocity field (arrows) for BIO and MIO configurations, respectively, obtained by finite element modeling (FEM) of fluid flow. (C, D) Fluid velocity in the plane $50 \mu \mathrm{m}$ above the cell culture surface (blue line) and shear stress along the centerline (circles) for the BIO and MIO configurations, respectively. Gray vertical lines show the boundary of the MBA well. (E, F) Spatial distributions of oxygen concentration (color map), fluid velocity (arrows) and velocity streamlines (lines) for the BIO and MIO configurations, respectively. (G) Calculated mass transport data for the BIO and MIO configurations, for two representative molecules: oxygen (as a small critical nutrient) and albumin (representative of a large growth factor). Data correspond to images (E, F).

characteristically high cell densities over 7 days of culture (Fig. 3A, a-c). Close inspection of the chamber outlet channel revealed higher cell density with respect to the inlet channel due to cell migration along the velocity field line (Fig. 3A, c).
This phenomenon was particularly noticeable within the $100 \mu \mathrm{m}$ high outlet channel section of the MBA-BIO, where the average velocity and shear stress are high $\left(\sim 100 \mu \mathrm{m} \mathrm{s}^{-1}\right.$ and $\sim 0.1 \mathrm{dyn} \mathrm{cm}^{-2}$ at the flow rate of $\left.10^{-6} \mathrm{ml} \mathrm{s}^{-1}\right)$. Within 
$\mathbf{A}$
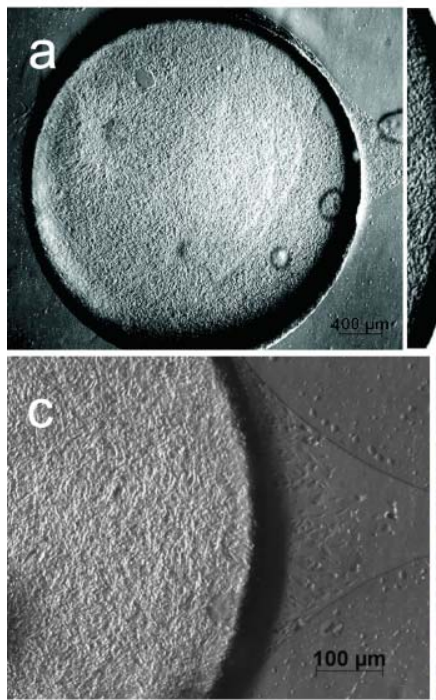

B
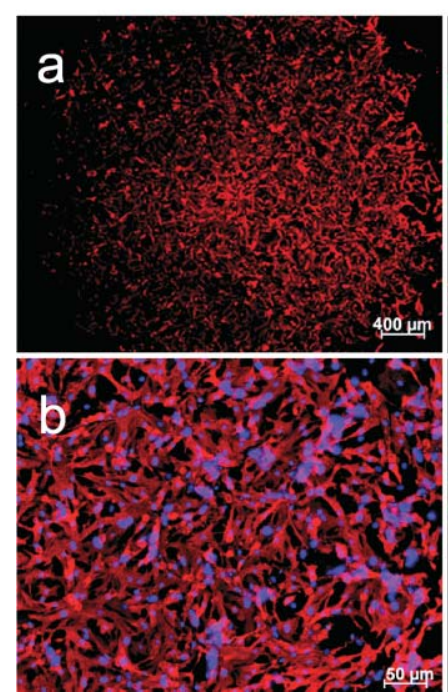

MBA BIO
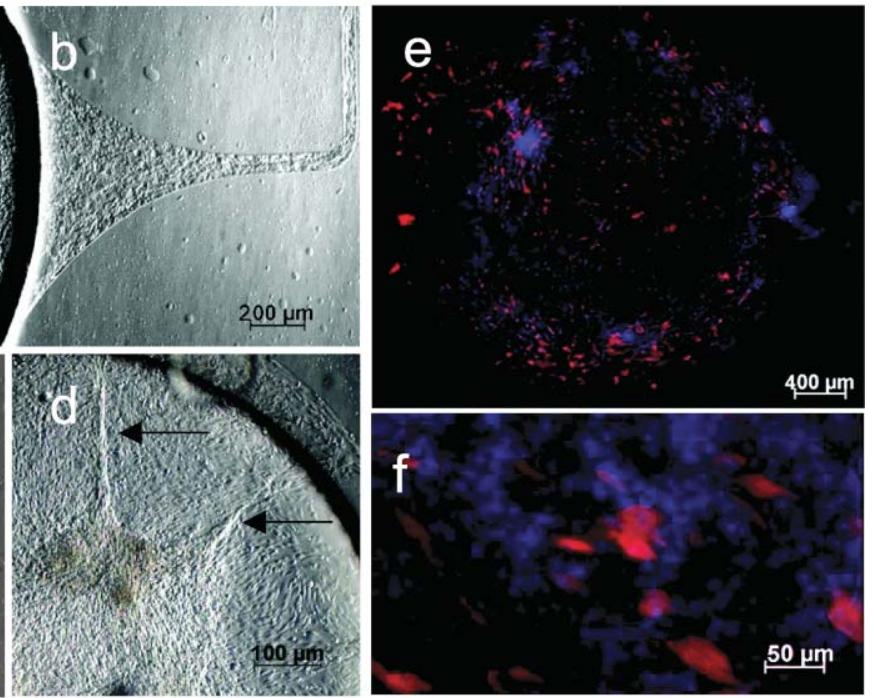

MBA MIO
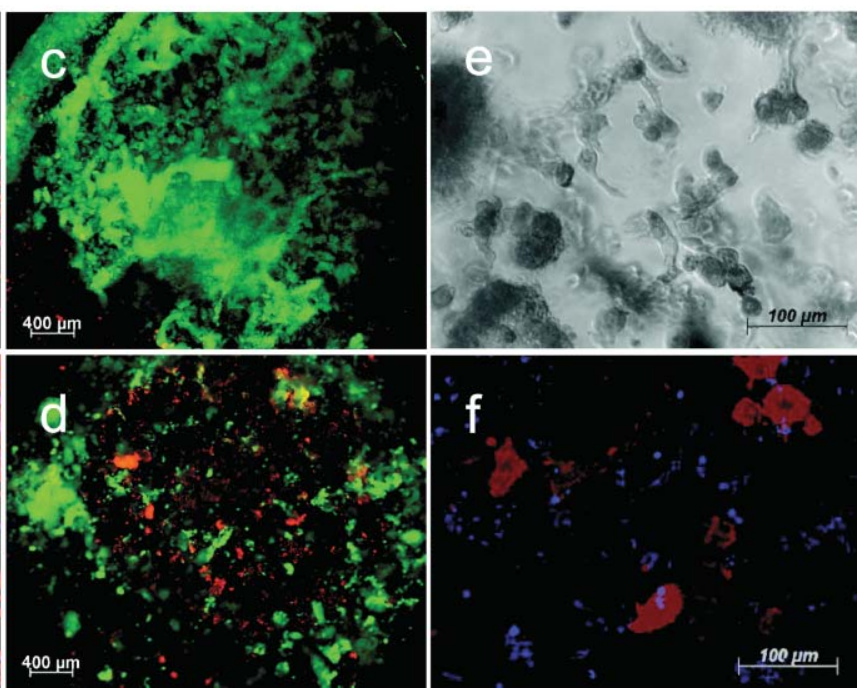

Fig. 3 Cultivation of three representative cell types (A) MBA-BIO system evaluation with $\mathrm{C} 2 \mathrm{C} 12$ cell line. (a) Phase contrast image showing homogenous distribution of $\mathrm{C} 2 \mathrm{C} 12$ over the whole surface area (7 days, MBA-BIO configuration); cells in the outlet channel are shown on the right. (b) Enlargement of the outlet channel of the chamber showing cell migration in the direction of fluid flow; only a few cells are present in the inlet section (not shown). (c) Spatially homogeneous cell distribution (higher magnification image). (d) Cell differentiation into multinucleated myotubes (arrows). (e) Immunostaining of $\mathrm{C} 2 \mathrm{C} 12$ for Tropomyosin in the entire well (shown in red; nuclei are shown in blue) in the entire well. (f) High magnification of the myotubes (Tropomyosin: red; nuclei: blue). (B) MBA-MIO system evaluation with primary neonatal rat cardiomyocytes and hESCs. (a) Fluorescent immunostaining for Troponin I showing homogenous cardiomyocytes distribution at 4 days within MBA-MIO configuration. (b) Higher magnification of Troponin I and DAPI immunostaining of cardiomyocytes. The staining shows the lack of differentiation of the cardyomyocyte during the MBA culture. (c, d) Representative images of hESCs (4 days), (c) with and (d) without perfusion of culture medium (green: live cells; red: dead cell nuclei). (e) Addition of $100 \mathrm{ng}$ hVEGF resulted in hESC sprouting and elongation outside the colonies (bright field image). (f) Sprouting cells expressed a-SMA, indicative of vascular differentiation (shown in red, confocal microscopy image).

7-10 days, cells formed multinucleated myotubes that were distributed throughout the culture well (Fig. 3A, d-e), elongated and expressed smooth muscle actin (Fig. 3A, e-f). Coating the chamber surfaces with collagen enabled neonatal rat cardiac myocytes to adhere and subsequently form spatially uniform monolayers (Fig. 3B, a-b). Cell viability, morphology and phenotype were well maintained, as seen by the expression of Troponin I. Spontaneous macroscopic contractions demonstrated that the cells were functionally active throughout the culture period.

The cultivation of hESCs turned out to be, as in most other systems, significantly more challenging than that observed for the other cell types. Similar to cardiac myocytes, hESCs required uniform collagen coating of the substrate to obtain even cell distribution (see Methods for details). The hESCs were also cultured encapsulated in a thin layer of hydrogel 
(hyaluronic acid, HA), a configuration that is $3 \mathrm{D}$ for the cells but effectively 2D from the perspective of the bioreactor operation.

For cultivation in a 2D setting (MIO), hESCs retained high viability with all conditions (data not shown). For cultivation in a $3 \mathrm{D}$ setting, medium perfusion affected the viability of all cells studied and most prominently the viability of hESCs (representative data for hESCs shown in Fig. 3B, c-d), presumably due to the combination of enhanced transport at the hydrogel surface and removal of any residual reagents from hydrogel polymerization. After 4 days of culture, the measured fractions of viable cells were $55 \pm 10$ and $67 \pm 65$ total cells for the static and perfused culture, respectively. To induce vascular differentiation of the HA-encapsulated hESCs, human vascular growth factor (hVEGF) was added to the culture medium, which resulted in extensive cell sprouting and $\alpha$-smooth muscle actin ( $\alpha$-SMA) expression (Fig. 3B, e-f).

Taken together, these studies demonstrated that both MBA configurations (BIO, MIO, Fig. 1) support the growth and differentiation of healthy and viable cells.

\section{Vascular differentiation of hESCs: effects of cell density and flow configuration on SMA expression}

The MBA system was designed for live imaging of culture wells with optical and fluorescent microscopy. To take advantage of this option in studies of hESCs, we developed a simple automated system for image analysis that allows fast and unbiased analysis of cell growth and differentiation. Automated and semi-automated routines have been used to measure other biological parameters, such as the spindle length in a Drosophila cell line. ${ }^{43}$ The routine is similar, but not identical (measurements of the length $v s$. the numbers of positive cells). Fig. 4A shows a representative image of hESCs differentiating into vascular lineages and labeled for $\alpha$-SMA (red), Oct4 (green) and DAPI (blue). A robust algorithm is used to assess the expression of nuclear and cytoplasmic markers in images of this kind.

To generate quantitative data, the positions of the individual cells stained with DAPI are first determined for a given view field. The expression of intranuclear (e.g., Oct4) and
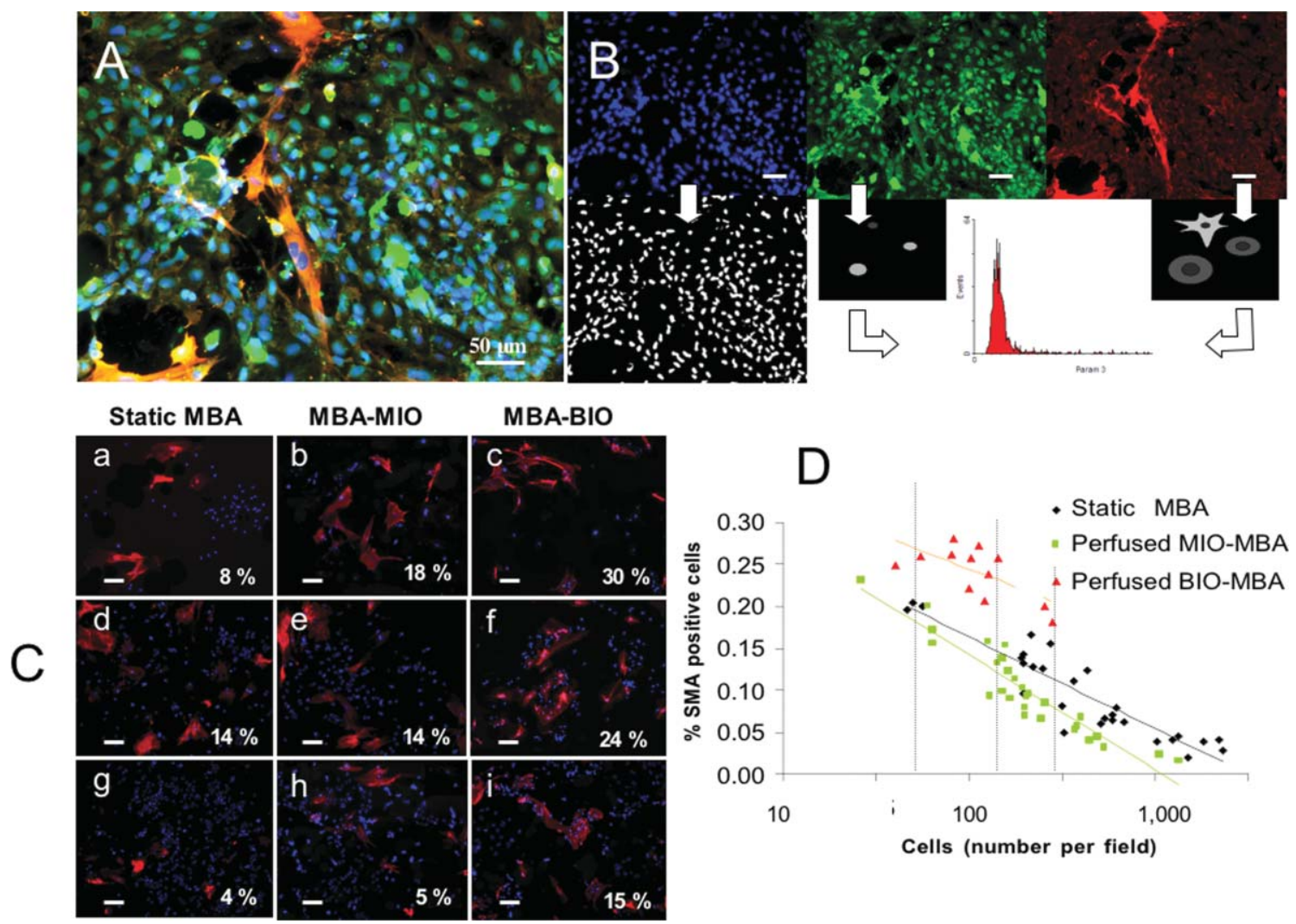

Fig. 4 Effects of cell density and flow configuration on hESC differentiation within the MBA device. (A) A representative merged image of cultured hESCs with immunofluorescent labels for $\alpha$-SMA (red), Oct4 (green) and DAPI (blue). (B) Automated image analysis of cell differentiation. Individual cells are identified by nuclear staining (DAPI) and the coordinates of nuclei are stored in a data matrix. The presence or absence of a nuclear label (Oct4 is shown as an example) and cytoplasmic label ( $\alpha$-SMA is shown as an example) is identified for each identified cell. Data are processed by a flow cytometer-like analysis, to obtain a dot plot or a histogram representing the intensity of a specific marker within the cell population. (C) Examples of the immunofluorescence of SMA (red) and DAPI (blue) for hESCs cultured in three different flow configurations: static MBA (left column: a, d, g), perfused MBA-MIO (middle column: b, e, h) and MBA-BIO (right column: c, f, i), three different cell densities: $60 \pm 6$ (top row: a, b, c), $160 \pm 4$ (middle row: d, e, f) and $314 \pm 15$ (bottom row: g, h, i). Scale bars: $200 \mu \mathrm{m}$. (D) The fraction of differentiated cells was determined using the automated image analysis for each flow configuration for a range of cell densities observed over the time of the culture. Data are plotted as the fraction of hESCs expressing $\alpha$-SMA as a function of the total number of cells in the field (an index of cell density) for each flow configuration. Vertical dotted lines correspond to the conditions in panels (C). 
cytoplasmic (e.g., $\alpha$-SMA) markers is then tracked for each individual cell, and represented in the form of a histogram or a dot plot in a manner common to flow cytometry. Fig. 4B shows a schematic description of the developed imaging routine, which was utilized to probe the effects of cell density and flow regime on hESC differentiation.

hESCs were cultured using the MBA-BIO and MBA-MIO configurations, and a non-perfused MBA-BIO to serve as a static control. Cell density varied from $3 \times 10^{2}$ to $3 \times$ $10^{4}$ cells $\mathrm{cm}^{-2}$, corresponding to $\sim 30$ to 3000 cells per well. Vascular differentiation was evaluated by the expression of $\alpha$-SMA, using the imaging routine described above.

Fig. 4C shows representative images of cells stained for $\alpha$-SMA (red) and DAPI (blue), that were cultured using three different flow configurations (static, BIO and MIO), The top, middle and bottom rows of panels show images obtained for low, medium and high cell density $(60 \pm 6,160 \pm 4$ and $314 \pm 15$ cells per well, respectively).

In general, cells cultured in the MBA-BIO configuration (the one with a higher level of shear) exhibited higher levels of differentiation relative to the other two configurations (Fig. 4 $\mathrm{C}, \mathrm{D})$. This is consistent with the known role of hydrodynamic shear in vascular differentiation. ${ }^{44}$

In all three configurations, the fraction of hESCs expressing $\alpha$-SMA increased as the cell density decreased (Fig. 4D). For example, in the MIO configuration, the percent of cells expressing $\alpha$-SMA decreased from $\sim 23 \%$ to $\sim 2 \%$ as the cell density increased from $\sim 200$ cells $\mathrm{cm}^{-2}$ to $\sim 10000$ cells $\mathrm{cm}^{-2}$. Cells cultured in the BIO configuration showed a similar trend, at higher expression levels of $\alpha$-SMA for comparable cell densities (Fig. 4D). Interestingly, Oct4 was not dependent on cellular density (data not shown). The fractional expression of the $\alpha$-SMA in hESC culture decreased linearly with the logarithm of cell density for all three bioreactor configurations. This graph summarizes the results from $\sim 70$ individual bioreactors for multiple MBA configurations. The automated assay for quantification of cell differentiation markers, presented here for the case of vascular differentiation of hESCs in 2D culture, could be extended to $3 \mathrm{D}$ cultures of cells and a variety of other differentiation markers.

This study demonstrated that the MBA could be used to study the effects of culture parameters on hESC differentiation, in a systematic manner and with only a minimal consumption of cells and reagents. The image processing routine used to assess the fractions of cells expressing differentiation markers (SMA and Oct4 in this case) can be considered an integral part of the proposed method.

\section{Application of MBA in studies of hESCs}

The MBA was designed to culture hESCs in a microarray setting, under controlled conditions, with imaging compatibility, and only minimal consumption of cells and reagents. hESCs are difficult to grow, require expensive medium and reagents, and from a biological perspective, their complex signaling networks and largely unknown regulatory factors often result in data variability. The use of a system that combines the advantages of microarrays (large numbers of groups and replicates, small volumes) and bioreactors (tight control of environmental conditions) with the automated routines of image processing to assess cell differentiation can largely improve the yield and quality of experimental data.

In general, the MBA shares many properties with other microfluidic-based platforms: low consumption of media, inexpensive fabrication, imaging compatibility, and high throughput. However, MBAs also provide close control of culture parameters (including hydrodynamic shear) in each culture well. Additional practical advantages include open access to the cell culture plane in each micro-bioreactor well, to ease surface coating, cell seeding and staining procedures. This allows for an easy translation of the existing biological protocols already established for hESCs to use in MBA formats, and a relatively straightforward use of existing liquid handling-robotic systems and microplate readers.

Notably, each MBA well maintains its own set of steadystate conditions via perfusion of culture medium in a singlepass mode, and without interaction with other wells within the same array. The medium at the outlet of each well is sent to the waste instead of being recirculated back to the cells (as in most perfused bioreactors). This way, there is no interaction between the wells, and the experiments are not confounded by paracrine signals being transferred downstream. In spite of single-pass flow, the consumption of medium is minimal because of the miniature size of the device. In studies of vascular differentiation of hESCs, 70 data points were generated over 4 days of culture using only $10 \mathrm{ml}$ of medium.

One of our goals was to provide a uniform and controllable hydrodynamic environment for all cells in the culture chamber. The CFD-assisted design yielded two different configurations-MBA-BIO and MBA-MIO — which exhibit hydrodynamic shear stress that can be as low as $<0.1$ and $<0.001 \mathrm{dyn}^{\mathrm{cm}}{ }^{-2}$, respectively. Both values are significantly below the threshold levels of shear stress that can affect the cells (1-5 dyn $\mathrm{cm}^{-2}$ ), a feature very useful for cultivation of hESCs in a quiescent regime. On the other hand, if hydrodynamic shear is needed (e.g., to mediate cell differentiation) these levels can be increased by a simple increase of fluid velocity. Also, the BIO and $\mathrm{MIO}$ configurations provide different flow environments, and the experiments described above show that the culture of hESCs in a BIO configuration resulted in larger values of $\alpha$-SMA-positive cells than culture in the MIO configuration.

\section{Methods}

\section{Fabrication of the MBA}

The micro-bioreactor array was designed in a standard CAD program (SolidWorks, Concord, MA, USA), and then exported to Adobe Illustrator 9.0 (San Jose, CA, USA) for printing as a transparency mask (5080 dpi, PageWorks, Cambridge, MA, USA). The mask was used to prepare a 4" silicon wafer-SU-8 2100 master of the bioreactor image. The processing guidelines of the photoresist manufacturer were followed to achieve features with a uniform height of $100 \mu \mathrm{m} .^{45}$

The actual bioreactor layers were replica molded ${ }^{46,47}$ by pouring $35 \mathrm{ml}$ of PDMS (Sylgard 184 Silicone Elastomer, Ellsworth Adhesives, Germantown, WA, USA) over a master 
placed in a $150 \mathrm{~mm}$ Petri dish. Prior to this step, a thin layer of hexamethyldisilane (Fluka, Buchs, Switzerland) was vacuum deposited on the wafer surface to prevent adherence of the PDMS. The fluidic connection ports were punched through the PDMS with a 21-gauge stainless steel tube $(0.635 \mathrm{~mm}$ ID, $0.81 \mathrm{~mm}$ OD, McMaster-Carr, Atlanta, GA, USA). In a similar manner, the micro-bioreactor wells were cored out using a $3.5 \mathrm{~mm}$ punch (Fray Products Corp., Buffalo, NY, USA), resulting in holes that were $3.35 \mathrm{~mm}$ in diameter. The layers of PDMS and glass were irreversibly bonded after treatment with vacuum gas plasma for $45 \mathrm{~s}$ (at $0.5 \mathrm{mbar}$ and $50 \mathrm{~W}$ ) (Harrick Scientific, Pleasantville, NY, USA). After coating the bottom surface of each well and seeding with cells, the entire bioreactor array was covered with a thin layer $(0.2 \mathrm{~mm})$ of PDMS. The MBA layers were held in place by mechanical compression via a frame consisting of an aluminium base, a polycarbonate cover, and four thumbscrews. Small lengths of stainless steel tubing (21-gauge, $10 \mathrm{~mm}$ length) were used to connect the MBA ports to soft Tygon tubing $(0.8 \mathrm{~mm}$ ID, $2.4 \mathrm{~mm}$ OD, Cole Palmer, Vernon Hills, IL, USA). The medium flow rate was controlled via a syringe pump, with an accuracy of $0.1 \mu 1 \mathrm{~min}^{-1}$ (PHD, Harvard Apparatus, Holliston, MA, USA). The culture medium was kept at $4{ }^{\circ} \mathrm{C}$, while the entire MBA device was kept in an incubator (NAPCO, Winchester VA, USA) at standard conditions $\left(37{ }^{\circ} \mathrm{C}, 95 \%\right.$ humidity, $\left.5 \% \mathrm{CO}_{2}\right)$.

\section{Hydrodynamic simulation}

The Navier-Stokes equations for incompressible fluids were solved using Comsol Multiphysics software (Burlington, MA, USA) to obtain a flow field within the micro-bioreactor well for the BIO and MIO configurations. The 3D domain of the micro-bioreactor well was meshed using four-node tetrahedron finite elements with a maximum mesh size of $300 \mu \mathrm{m}$. Coarsening and refining of the mesh space grid ensured solutions independent of the spatial discretization.

The inlet boundary condition was set for a fully developed velocity profile (calculated on the basis of flow rate), while a zero pressure condition was imposed on the outlet and non-slip boundary condition set for channel and bioreactor walls. Assumption of longitudinal symmetry allowed solving the equations for only one half of the channel and chamber, thus minimizing computational time.

The medium viscosity and density values for DMEM medium with $5 \%$ FCS were taken from the literature. ${ }^{48}$ As a check on the validity of the simulation, the shear stress within the uniform rectangular channels was calculated from an approximation of the analytical solution for flow between parallel plates, $\tau=6 \mu Q / b h^{2}$ where $\mu$ is the dynamic viscosity of the medium $\left(0.0077 \mathrm{dyn} \mathrm{s} \mathrm{cm}^{-2}\right), Q$ is the flow rate $\left(5 \times 10^{-6} \mathrm{ml} \mathrm{s}^{-1}\right), b$ is the channel width $(0.01 \mathrm{~cm})$, and $h$ is the channel height $(0.01 \mathrm{~cm})$. The calculated value of 0.058 dyn $\mathrm{cm}^{-2}$ compares well to the result obtained by finite element modeling of the flow in the channel $\left(\sim 0.07 \mathrm{dyn} \mathrm{cm}^{-2}\right.$ at the position $0.32 \mathrm{~cm}$ from center of the well, Fig. 2C).

\section{Mass transport calculations}

To obtain concentration profiles within the micro-bioreactor wells for the BIO and MIO configurations, the mass balance equations for a convective diffusive regime were solved using Comsol Multiphysics software (Burlington, MA, USA). Fluid velocity profiles were obtained from the three dimensional solution. The concentration of $1 \mathrm{mM}$ was used as a boundary condition at the inlet; zero concentration was imposed as a boundary condition at the cell surface. The $R e$ and $P e$ numbers were then calculated using standard equations and literature values of diffusion coefficients for each solute.

\section{Cell culture}

C2C12 myoblast cells (murine, American Type Culture Collection) were grown to confluency in growth medium (DMEM with 20\% fetal bovine serum, FBS). Cells were trypsinized, counted, and 2000 cells were added to each MBA well. After $12 \mathrm{~h}$, medium perfusion was initiated at a flow rate of $0.3 \mu \mathrm{l} \mathrm{min}{ }^{-1}\left(0.432 \mathrm{ml} \mathrm{day}{ }^{-1}\right)$, which corresponds to $0.075 \mu \mathrm{l} \mathrm{min}{ }^{-1}$ per bioreactor. Perfusion medium contained low serum levels ( $2 \% \mathrm{FBS})$, to induce myogenic differentiation. Experimental methods with primary cells (cardiomyocytes) were similar to those with $\mathrm{C} 2 \mathrm{C} 12$ line except that cells were seeded at 1000 cells per well and the culture medium contained $10 \%$ FBS. Details on cardiomyocyte isolation from 2-day old neonatal Sprague Dawley rats can be found elsewhere. ${ }^{49}$

Non-differentiated hESCs (lines H9 and H13; passage 20-35) (WiCell Research Institute, Madison, WI, USA) were grown on inactivated mouse embryonic fibroblasts (Chemicon International, Temecula, CA, USA) in growth medium consisting of $80 \%$ KnockOut DMEM, supplemented with $20 \%$ KnockOut Serum replacement, $4 \mathrm{ng} \mathrm{ml}^{-1}$ basic Fibroblast Growth Factor, $1 \mathrm{mM}$ L-glutamine, $0.1 \mathrm{mM} \beta$-mercaptoethanol, and $1 \%$ non-essential amino acid stock (all from Invitrogen Corp., Carlsbad, CA, USA). hESCs were passaged to a new feeder layer using $1 \mathrm{mg} \mathrm{ml}^{-1}$ type IV collagenase (Invitrogen Corp., Carlsbad, CA, USA). For 3D studies, hESCs were removed from the feeder layer by incubation with collagenase for 20-30 min. For 2D studies, hESCs were removed from MEFs by incubation with EDTA (Promega), with $5 \% \mathrm{FCS}$, for $20 \mathrm{~min}$, followed by separation into single cells using a $40 \mu \mathrm{m}$ mesh strainer (Falcon). For 2D studies, the glass surface of each MBA chamber was treated with a solution of 4\% 3-aminopropyltrimethoxysilane (SigmaAldrich, St. Louis, MO, USA) in acetone for $15 \mathrm{~min}$. Each well was then coated with $0.05 \mathrm{mg} \mathrm{ml}^{-1}$ collagen IV (R\&D Systems, Minneapolis, MN, USA) for $1 \mathrm{~h}$ at room temp. The cells were seeded at concentrations of $10^{3}, 3 \times 10^{3}, 5 \times 10^{3}$, and $10^{4}$ cells per well. Medium perfusion was started $24 \mathrm{~h}$ after cell seeding to allow sufficient time for cell attachment.

\section{Hydrogel preparation and hESC microencapsulation}

For 3D studies, we utilized previously developed photocrosslinkable methacrylated HA hydrogels. ${ }^{50-52}$ We recently showed that these HA hydrogels support the propagation of undifferentiated hESCs and the initiation of vascular differentiation. ${ }^{53}$ For hydrogel formation, methacrylated HA was dissolved in PBS containing 0.05 wt\% 2-methyl-1-[4-(hydroxyethoxy)phenyl]-2-methyl-1-propanone (Irgacure 2959, I2959) and exposed to ultraviolet light (Black Ray, Redding, CA, USA) for $10 \mathrm{~min}$. These encapsulation conditions have 
previously been used for the encapsulation of a variety of mammalian cells. ${ }^{54}$

hESCs were added to the precursor solution at a concentra-

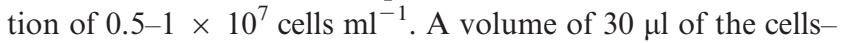
HA mixture was poured into each micro-bioreactor well, and photopolymerized (using $\sim 10 \mathrm{~mW} \mathrm{~cm}^{-2} \mathrm{UV}$ light, BlackRay) for $10 \mathrm{~min}$. Cell-gel constructs were cultivated in growth medium (for proliferation), and endothelial cell medium (for differentiation) (PromoCell, Heidelberg, Germany). In some studies, medium was supplemented with $100 \mathrm{ng} \mathrm{ml}^{-1} \mathrm{hVEGF}$ (R\&D systems, Minneapolis, MN, USA).

\section{Immunofluorescence}

Cells were fixed in situ with accustain (Sigma-Aldrich, St. Louis, MO, USA) for $25 \mathrm{~min}$ at room temperature. Cells were stained for $1 \mathrm{~h}$ with the first antibody and then for $30 \mathrm{~min}$ with a secondary antibody, at room temperature. The wells were rinsed three times with PBS without calcium and magnesium (Invitrogen Corp., Carlsbad, CA, USA). DAPI (2 $\mu \mathrm{g} \mathrm{ml}^{-1}$; Sigma-Aldrich) was added to the last rinse. C2C12 cells were stained for monoclonal Tropomyosin (Sigma-Aldrich). hESCs were stained either for smooth muscle actin (1:20; Dako, Troy Michigan, USA), or Oct4 (1 : 50; R\&D Systems, Minneapolis, $\mathrm{MN}$, USA). For secondary staining, Cy3 or the FITCconjugated antibody (1 : 50; Sigma-Aldrich, St. Louis, MO, USA) were used. Cardiomyocytes were stained for anti-rabbit Troponin I and the FITC-conjugated secondary antibody (both from Chemicon International, Temecula, CA, USA). The immuno-labeled cells were examined using a fluorescence microscope (Axiomat, Zeiss, Thornwood, NY, USA).

\section{Data acquisition and analysis}

The percent of differentiated and undifferentiated cells were evaluated by an imaging acquisition program written using scripts available in MATLAB, and MATLAB imaging toolbox (The MathWorks, Natick, MA, USA). Images of the entire micro-bioreactor well area $(3.5 \mathrm{~mm}$ diameter) were acquired as a series of images at $5 \times$ magnification for the nuclei (DAPI) and two other markers (cytoplasmic and nuclear intracellular markers). From the DAPI image, the coordinates of the pixel of each nucleus were acquired and stored. The intensity of the pixels corresponding to the DAPI coordinates in the other two images was used to evaluate the existence of nuclear transcription factors. The intensity of the cytoplasmic staining fluorescence was evaluated by considering the intensity of pixels within the ellipsoid corona centered in the nucleus and an area $50 \%$ bigger in size. Processed data were then used to count the number of nuclei and evaluate the fraction of cells expressing a specific marker. Graphical representation of data (dot plots, histograms) and statistical evaluation of signal intensities were performed by importing data into a free flow cytometry data analysis program (WinMDI 2.8).

\section{Conclusions}

Stem cell biology is increasingly relying on advanced technologies that provide better cell culture microenvironments and enable control over multiple molecular and physical regulatory signals. These enabling technologies are of particular interest to hESCs, because of the complexity of their regulatory pathways, and uncontrolled variables associated with traditional culture methods. We have developed a microbioreactor array that combines the advantages of microarrays with those of bioreactors, and provides a means to study the growth and differentiation of hESCs under controlled conditions and in a multiarray setting. The device is the size of a microscope slide and contains twelve independent microbioreactors perfused with culture medium. The design is flexible, as it allows $2 \mathrm{D}$ and $3 \mathrm{D}$ cell culture under variable levels of hydrodynamic shear and mass transport, which were characterized by computational methods. To demonstrate the operation of the MBA, we cultured $\mathrm{C} 2 \mathrm{C} 12$ cells, rat cardiac myocytes and hESCs within the system. A system for automated in situ image analysis of the expression of cell differentiation markers has also been developed and used to investigate the effects of cell density and flow regime on vascular differentiation of hESCs. This device can thus serve as a tool for studying hESCs in the context of their environment.

\section{Acknowledgements}

Authors would like to acknowledge support of the NIH (P41 EB002520 and R01 HL076485 to GV-N), CaRiPaRo (PhD grant to EF), JDRF (fellowship to $\mathrm{SG}$, innovative grant 5-2005-1106 to GV-N), Fulbright Commission (Fellowship to NE), and the University of Padova (Progetti di Ateneo).

\section{References}

1 F. M. Watt and B. L. M. Hogan, Science, 2000, 287, 1427.

2 K. Powell, Nature, 2005, 435, 268.

3 R. Schofield, Biomed. Pharmacother., 1983, 37, 375.

4 L. Jones, Curr. Biol., 2001, 11, R484.

5 E. Fuchs, T. Tumbar and G. Guasch, Cell, 2004, 116, 769.

6 L. G. Griffith and G. Naughton, Science, 2002, 295, 1009.

7 S. R. Khetani and S. N. Bhatia, Curr. Opin. Biotechnol., 2006, 17, 524.

8 J. M. Polak and A. E. Bishop, Ann. N.Y. Acad. Sci., 2006, 1068, 352.

9 R. Portner, S. Nagel-Heyer, C. Goepfert, P. Adamietz and N. Meenen, J. Biosci. Bioeng., 2005, 100, 235.

10 G. Vunjak-Novakovic, L. Meinel, G. Altman and D. Kaplan, Orthod. Craniofac. Res., 2005, 8, 209.

11 M. Horner, W. M. Miller, J. M. Ottino and E. T. Papoutsakis, Biotechnol. Prog., 1998, 14, 689.

12 M. R. Koller, J. G. Bender, W. M. Miller and E. T. Papoutsakis, Nat. Biotechnol., 1993, 11, 358.

13 T. Dvir, N. Benishti, M. Shachar and S. Cohen, Tissue Eng., 2006, 12, 2843.

14 W. J. Fong, H. L. Tan, A. Choo and S. K. Oh, Bioprocess Biosyst. Eng., 2005, 27, 381.

15 S. M. Dang, S. Gerecht-Nir, J. Chen, J. Itskovitz-Eldor and P. W. Zandstra, Stem Cells, 2004, 22, 275.

16 S. Gerecht-Nir, S. Cohen and J. Itskovitz-Eldor, Biotechnol. Bioeng, .2004, 86, 493.

17 H. Andersson and A. Van Den Berg, Lab Chip, 2004, 4, 98.

18 D. J. Beebe, G. A. Mensing and G. M. Walker, Ann. Rev. Biomed. Eng., 2002, 4, 261.

19 V. Chin, P. Taupin, S. Sanga, J. Scheel, F. H. Gage and S. N. Bhatia, Biotechnol. Bioeng., 2004, 88, 399.

20 A. Folch, B. Jo, O. Hurtado, D. Beebe and M. Toner, J. Biomed. Mater. Res., 2000, 52, 346.

21 A. Khademhosseini, J. Yeh, G. Eng, J. Karp, H. Kaji, J. Borenstein, O. C. Farokhzad and R. Langer, Lab Chip, 2005, 5, 1380 . 
22 D. G. Anderson, S. Levenberg and R. Langer, Nat. Biotechnol., 2004, 22, 863.

23 N. Futai, W. Gu and T. S. J. W. Song, Lab Chip, 2006, 6, 149.

24 D. R. Albrecht, G. H. Underhill, T. B. Wassermann, R. L. Sah and S. N. Bhatia, Nat. Meth., 2006, 3, 369.

25 G. M. Whitesides, E. Ostuni, S. Takayama, X. Jiang and D. E. Ingber, Ann. Rev. Biomed. Eng., 2001, 3, 335.

26 J.-W. Choi, Fabrication of Micromachined Magnetic Particle Separators for Bioseparation in Microfluidic Systems. Microfluidic Techniques: Reviews and Protocols, 2005, pp. 65-82.

27 X. Zhang, J. M. Cooper, P. B. Monaghan and S. J. Haswell, Lab Chip, 2006, 6, 561.

28 S. N. Bhatia, M. L. Yarmush and M. Toner, J. Biomed. Mater. Res., 1997, 34, 189.

29 R. S. Kane, S. Takayama, E. Ostuni, D. E. Ingber and G. M. Whitesides, Biomaterials, 1999, 20, 2363.

30 M. A. McClain, C. T. Culbertson, S. C. Jacobson, N. L. Allbritton, C. E. Sims and J. M. Ramsey, Anal. Chem., 2003, 75, 5646.

31 J. El-Ali, P. K. Sorger and K. F. Jensen, Nature, 2006, 442, 403.

32 G. M. Whitesides, Nature, 2006, 442, 368.

33 P. Ales, P. Zdenka, S. David, K. Eugene, W. John, C. David and B. Franz, Biomed. Microdev., 2004, V6, 325.

34 L. Kim, M. Vahey, H. Lee and J. Voldman, Lab Chip, 2006, 6, 394

35 Jaesung Park, F. Berthiaume, M. Toner, M. L. Yarmush and A. W. Tilles, Biotechnol. Bioeng., 2005, 90, 632.

36 D. M. Thompson, K. R. King, K. J. Wieder, M. Toner, M. L. Yarmush and A. Jayaraman, Anal. Chem., 2004, 76, 4098.

37 Y.-C. Toh, C. Zhang, J. Zhang, Y. M. Khong, S. Chang, V. D. Samper, D. v. Noort, D. W. Hutmacher and H. Yu, Lab Chip, 2007, 7, 302.

38 M. J. Powers, K. Domansky, M. R. Kaazempur-Mofrad, A. Kalezi, A. Capitano, A. Upadhyaya, P. Kurzawski, K. E. Wack, D. Beer Stolz, R. Kamm and L. G. Griffith, Biotechnol. Bioeng., 2002, 78, 257.
39 D. Vandendriesche et al., J. Gravit. Physiol., 2004, 11, 93.

40 M. Radisic, H. Park, H. Shing, T. Consi, F. J. Schoen, R. Langer, L. E. Freed and G. Vunjak-Novakovic, Proc. Natl. Acad. Sci. U. S. A., 2004, 101, 18129.

41 S. Gerecht-Nir, A. Ziskind, S. Cohen and J. Itskovitz-Eldor, Lab. Invest., 83, 1811.

42 S. C. A. Z. J. I.-E. Sharon Gerecht-Nir, Biotechnol. Bioeng., 2004, 88, 313 .

43 G. Goshima, R. Wollman, N. Stuurman, J. M. Scholey and R. D. Vale, Curr. Biol., 2005, 15, 1979.

44 H. Huang et al., J. Artif. Organs, 2005, 8, 110.

45 MicroChem_Corp., www.microchem.com, 2006.

46 R. J. Jackman, S. T. Brittain, A. Adams, M. G. Prentiss and G. M. Whitesides, Science, 1998, 280, 2089.

47 J. C. McDonald and G. M. Whitesides, Acc. Chem. Res., 2002, 35, 491.

48 J. L. Moreira, P. C. Santana, A. S. Feliciano, P. E. Cruz, A. J. Racher, J. B. Griffiths and M. J. Carrondo, Biotechnol. Prog., $1995, \mathbf{1 1}, 575$

49 C. Cannizzaro, N. Tandon, E. Figallo, H. Park, S. Gerecht, M. Radisic, N. Elvassore and G. Vunjak-Novakovic, Practical aspects of cardiac tissue engineering with electrical stimulation, Methods in molecular medicine: Tissue engineering, ed. H. Hauser and M. Fussenegger, Humana Press, 2007, pp. 291-307.

50 K. Smeds and M. Grinstaff, J. Biomed. Mater. Res., 2001, 54, 115.

51 C. Chung, J. Mesa, G. J. Miller, M. A. Randolph, T. J. Gill and J. A. Burdick, Tissue Eng., 2006, 12, 2665.

52 C. Chung, J. Mesa, M. A. Randolph, M. Yaremchuk and J. A. Burdick, J. Biomed. Mater. Res., 2006, 77A, 518.

53 S. Gerecht, J. A. Burdick, L. S. Ferreira, S. A. Townsend, R. Langer and G. Vunjak-Novakovic, Proc. Natl. Acad. Sci. U. S. A., 2007, in press.

54 S. J. Bryant, C. R. Nuttelman and K. S. Anseth, J. Biomater. Sci. Polym. E., 2000, 11, 439. 